\title{
Distributed Quasi-Nonlinear Model Predictive Control by Dual Decomposition
}

\author{
Alexandra Grancharova* Tor A. Johansen** \\ * Institute of System Engineering and Robotics, Bulgarian Academy of Sciences, Acad. G. Bonchev str., Bl.2, P.O.Box 79, \\ Sofia 1113, Bulgaria (Tel: +359889625010; e-mail: alexandra.grancharova@abv.bg) \\ ** Department of Engineering Cybernetics, Norwegian University of Science and Technology, 7491 Trondheim, Norway \\ (e-mail:Tor.Arne.Johansen@itk.ntnu.no)
}

\begin{abstract}
A suboptimal approach to distributed NMPC for a class of systems consisting of nonlinear subsystems with linearly coupled dynamics subject to both state and input constraints is proposed. The approach applies a dual decomposition method to represent the original centralized NMPC problem into a distributed quasi-NMPC problem by linearization of the nonlinear system dynamics and taking into account the couplings between the subsystems.
\end{abstract}

Keywords: Predictive control, Distributed control, Decomposition methods, Interconnected systems, Nonlinear systems, Constraints, Bounded disturbances.

\section{INTRODUCTION}

Model predictive control (MPC) has become the accepted methodology to solve complex control problems related to process industries. MPC involves the solution at each sampling instant of a finite horizon optimal control problem subject to the system dynamics, and state and input constraints. However, solving in a centralized way nonlinear MPC problems for medium- and large-scale systems may be impractical due to the complexity of the Nonlinear Programming (NLP) problem, the topology of the plant and data communication, and the large number of decision variables. Therefore, there is a strong motivation for development of methods for distributed solution of NMPC problems. At the same time, the massive multi-core computer architectures available nowadays would encourage parallel and distributed NMPC computations (Constantinides (2009)). Recently, several approaches for decentralized implementation of MPC algorithms have been developed, Scattolini (2009). As it is pointed out in Magni and Scattolini (2006), the possibility to use MPC in a decentralized fashion has the advantage to reduce the original, large size, optimization problem into a number of smaller and more tractable ones. In Venkat et al. (2004), Kim and Sugie (2005), Zhang and Li (2007), Giselsson and Rantzer (2010), approaches for distributed MPC for systems consisting of linear interconnected subsystems have been developed. The approach in Giselsson and Rantzer (2010) is based on the dual decomposition methods (Arrow et al. (1958), Dantzig and Wolfe (1961), Cohen and Miara (1990)), where large-scale optimization problems are handled by using Lagrange multipliers to relax the couplings between the sub-problems. In Rantzer (2007), Rantzer (2009), the dual decomposition is used for analysis and synthesis of distributed feedback controllers.

Further, approaches for distributed MPC for systems composed of several nonlinear subsystems have been proposed in Magni and Scattolini (2006), Dunbar and Murray
(2006), Keviczky et al. (2006). In Magni and Scattolini (2006), a stabilizing decentralized MPC algorithm for nonlinear systems consisting of several interconnected local subsystems is developed. It is derived under the main assumptions that no information can be exchanged between local control laws, i.e. the coupling between the subsystems is ignored, and only input constraints are imposed on the system. In Dunbar and Murray (2006), it is supposed that the dynamics and constraints of the nonlinear subsystems are decoupled, but their state vectors are coupled in a single cost function of a finite horizon optimal control problem. In Keviczky et al. (2006), an optimal control problem for a set of dynamically decoupled nonlinear systems, where the cost function and constraints couple the dynamical behavior of the systems, is solved.

In this paper, a suboptimal approach to distributed NMPC for a more general class of systems consisting of nonlinear subsystems with coupled dynamics subject to both state and input constraints is proposed. Like in Magni and Scattolini (2006), it is supposed that the couplings between the subsystems are linear. However in difference to Magni and Scattolini (2006), the distributed NMPC method proposed here takes into account these couplings, as well as state constraints. The suggested approach applies the dynamic dual decomposition method (Cohen and Miara (1990), Rantzer (2009), Giselsson and Rantzer (2010)) and reformulates the original centralized NMPC problem into a distributed quasiNMPC problem by linearization of the nonlinear system dynamics. The approach is based entirely on distributed online optimization (by gradient iterations) and can be applied to large-scale nonlinear systems.

\section{FORMULATION OF NONLINEAR MODEL PREDICTIVE CONTROL PROBLEM}

Consider a system composed by the interconnection of $M$ subsystems described by the following nonlinear discrete-time models (Magni and Scattolini (2006)): 
$x_{i}(t+1)=f_{i}\left(x_{i}(t), u_{i}(t)\right)+g_{i}(x(t))+d_{i}(t), i=1,2, \ldots, M$

where $x_{i}(t) \in \mathbb{R}^{n_{i}}, u_{i}(t) \in \mathbb{R}^{m_{i}}$, and $d_{i}(t) \in \mathbb{R}^{n_{i}}$ are the state, control input, and disturbance vectors, related to the $i$-th subsystem, and $f_{i}: \mathbb{R}^{n_{i}} \times \mathbb{R}^{m_{i}} \rightarrow \mathbb{R}^{n_{i}}$ and $g_{i}: \mathbb{R}^{n} \rightarrow \mathbb{R}^{n_{i}}$ are nonlinear functions. In (1), the mutual influence of the $M$ subsystems is described by the functions $g_{i}$, which depend on the overall state:

$$
x(t)=\left[x_{1}(t), x_{2}(t), \ldots, x_{M}(t)\right] \in \mathbb{R}^{n}, n=\sum_{i=1}^{M} n_{i}
$$

Similarly, the overall control input is denoted:

$$
u(t)=\left[u_{1}(t), u_{2}(t), \ldots, u_{M}(t)\right] \in \mathbb{R}^{m}, m=\sum_{i=1}^{M} m_{i}
$$

The following constraints are imposed on the subsystems:

$$
u_{\min , i} \leq u_{i}(t) \leq u_{\max , i}, x_{\min , i} \leq x_{i}(t) \leq x_{\max , i}, i=1,2, \ldots, M
$$

The following assumptions are made:

A1. The functions $f_{i}$ and $g_{i}, i=1, \ldots, M$ are $C^{1}$ functions with $f_{i}(0,0)=0, g_{i}(0)=0$.

A2. $x_{\min , i}<0<x_{\max , i}, u_{\min , i}<0<u_{\max , i}, i=1, \ldots, M$.

A3. The disturbances $d_{i}, i=1, \ldots, M$ are bounded by:

$$
\left|d_{i}(t)\right| \leq d_{\text {max }, i}, i=1,2, \ldots, M
$$

with $\quad d_{\text {max }, i} \in \mathbb{R}_{>0}^{n_{i}}, \quad d_{\max , i}<\left|x_{\min , i}\right| \quad$ and $\quad d_{\max , i}<x_{\max , i}$, $i=1,2, \ldots, M$, where the operation $|\cdot|$ is taken element-wise. It is supposed that a full measurement $x=\left[x_{1}, x_{2}, \ldots, x_{M}\right]$ of the overall state is available at the current time $t$. The optimal regulation problem is considered where the goal is to steer the overall state of the system (1) to the origin. For the current $x=\left[x_{1}, x_{2}, \ldots, x_{M}\right]$, the regulation NMPC solves the optimization problem:

\section{Problem P1 (Centralized NMPC):}

$$
V^{\text {opt }}(x)=\min _{U} J(U, x)
$$

subject to $x_{t \mid t}=x$ and:

$$
\begin{aligned}
& x_{i, t+k \mid t} \in \mathcal{X}_{i}, i=1, \ldots, M, k=1, \ldots, N-1 \\
& u_{i, t+k} \in \mathcal{U}_{i}, i=1, \ldots, M, k=0,1, \ldots, N-1 \\
& x_{i, t+k+1 \mid t}=f_{i}\left(x_{i, t+k \mid t}, u_{i, t+k}\right)+g_{i}\left(x_{t+k \mid t}\right) \\
& i=1, \ldots, M, k=0,1, \ldots, N-2 \\
& x_{t+k \mid t}=\left[x_{1, t+k \mid t}, x_{2, t+k \mid t}, \ldots, x_{M, t+k \mid t}\right], k=0,1, \ldots, N-1 \\
& u_{t+k}=\left[u_{1, t+k}, u_{2, t+k}, \ldots, u_{M, t+k}\right], k=0,1, \ldots, N-1
\end{aligned}
$$

with $U=\left[u_{t}, u_{t+1}, \ldots, u_{t+N-1}\right]$ and the cost function given by:

$$
J(U, x)=\sum_{k=0}^{N-1} \sum_{i=1}^{M} l_{i}\left(x_{i, t+k \mid t}, u_{i, t+k}\right)
$$

Here, $l_{i}\left(x_{i, t+k \mid t}, u_{i, t+k}\right)=\left\|x_{i, t+k \mid t}\right\|_{Q_{i}}^{2}+\left\|u_{i, t+k}\right\|_{R_{i}}^{2}$ is the stage cost for the $i$-th subsystem with weighting matrices $Q_{i}, R_{i} \succ 0$, and $N$ is a finite horizon. The sets $\mathcal{X}_{i}$ and $\mathcal{U}_{i}$ are defined by:

$$
\begin{aligned}
& \mathcal{X}_{i}=\left\{\lambda_{i} \in \mathbb{R}^{n_{i}} \mid x_{\text {min }, i}+d_{\text {max }, i} \leq \lambda_{i} \leq x_{\text {max }, i}-d_{\text {max }, i}\right\} \\
& \mathcal{U}_{i}=\left\{\eta_{i} \in \mathbb{R}^{m_{i}} \mid u_{\text {min }, i} \leq \eta_{i} \leq u_{\text {max }, i}\right\}
\end{aligned}
$$

It follows from (13)-(14) that $\mathcal{X}_{i}$ and $\mathcal{U}_{i}$ are convex (polyhedral) sets, which include the origin in their interior (due to Assumptions A2 and A3).
It should be noted that the state constraints (7) with the admissible set $\mathcal{X}_{i}$ defined by (13) guarantee the robust feasibility of the solution in sense that the original state constraints (4) will be satisfied for the worst-case disturbances.

\section{DISTRIBUTED QUASI-NMPC FOR NONLINEAR SYSTEMS WITH LINEAR COUPLINGS}

\subsection{Distributed NMPC by dual decomposition}

Problem P1 can be decomposed by using the dynamic dual decomposition approach (Cohen and Miara (1990), Rantzer (2009)). First, the following assumption is made:

A4. The functions $g_{i}(x(t))$ have the form:

$$
g_{i}(x(t))=\sum_{j=1, j \neq i}^{M} A_{i j} x_{j}(t), i=1, \ldots, M
$$

where $A_{i j} \in \mathbb{R}^{n_{i} \times n_{j}}$ are constant matrices.

The following decoupled state equations can be formulated:

$$
x_{i}(t+1)=f_{i}\left(x_{i}(t), u_{i}(t)\right)+v_{i}(t)+d_{i}(t), i=1, \ldots, M
$$

with the additional constraints that (Rantzer (2009)):

$$
v_{i}(t)=\sum_{j=1, j \neq i}^{M} A_{i j} x_{j}(t), i=1, \ldots, M \text { for all } t
$$

The variable $v_{i} \in \mathbb{R}^{n_{i}}$ can be interpreted as the influence of the other subsystems in the update of $x_{i}$. Then, similar to (Rantzer (2009)), the constraints (17) are relaxed by introducing the corresponding Lagrange multipliers $p_{i} \in \mathbb{R}^{n_{i}}$ in the cost function (12) and the problem P1 is reformulated as a distributed NMPC problem:

\section{Problem P2 (Distributed NMPC):}

$V^{\text {opt }}(x)=$

$$
\begin{aligned}
& \max _{P} \min _{U, X, V} \sum_{k=0}^{N-1} \sum_{i=1}^{M}\left[l_{i}\left(x_{i, t+k \mid t}, u_{i, t+k}\right)+p_{i, t+k}^{T}\left(v_{i, t+k}-\sum_{\substack{j=1 \\
j \neq i}}^{M} A_{i j} x_{j, t+k \mid t}\right)\right]= \\
& \max _{P} \sum_{i=1}^{M}\left(\min _{U_{i}, X_{i}, V_{i}} \sum_{k=0}^{N-1}[\underbrace{\left.\left.l_{i}\left(x_{i, t+k \mid t}, u_{i, t+k}\right)+p_{i, t+k}^{T} v_{i, t+k}-x_{i, t+k \mid t}^{T} \sum_{\substack{j=1 \\
j \neq i}}^{M} A_{j i}^{T} p_{j, t+k}\right]\right)}_{l_{i}^{P}\left(x_{i, t+k \mid t}, u_{i, t+k}, v_{i, t+k}, P\right)}\right.
\end{aligned}
$$

subject to $x_{t \mid t}=x$, constraints (7)-(8) and:

$$
\begin{aligned}
& x_{i, t+k+1 \mid t}=f_{i}\left(x_{i, t+k \mid t}, u_{i, t+k}\right)+v_{i, t+k} \\
& i=1, \ldots, M, k=0,1, \ldots, N-2 \\
& p_{t+N-1}=0
\end{aligned}
$$

Here:

$$
\begin{gathered}
P=\left[p_{t}, p_{t+1}, \ldots, p_{t+N-1}\right] \\
\text { with } p_{t+k}=\left[p_{1, t+k}, p_{2, t+k}, \ldots, p_{M, t+k}\right], k=0,1, \ldots, N-1 \\
U=\left[u_{t}, u_{t+1}, \ldots, u_{t+N-1}\right], U_{i}=\left[u_{i, t}, u_{i, t+1}, \ldots, u_{i, t+N-1}\right] \\
X=\left[x_{t \mid t}, x_{t+1 \mid t}, \ldots, x_{t+N-1 \mid t}\right], X_{i}=\left[x_{i, t \mid t}, x_{i, t+1 \mid t}, \ldots, x_{i, t+N-1 \mid t}\right] \\
V=\left[v_{t}, v_{t+1}, \ldots, v_{t+N-1}\right], V_{i}=\left[v_{i, t}, v_{i, t+1}, \ldots, v_{i, t+N-1}\right] \\
\text { with } v_{t+k}=\left[v_{1, t+k}, v_{2, t+k}, \ldots, v_{M, t+k}\right], k=0,1, \ldots, N-1
\end{gathered}
$$


The Lagrange multipliers $P$ are also referred to as prices (Rantzer (2009)) and the problem P2 can be interpreted as a game with two players for each subsystem. Given the prices, the objective of the first player for the $i$-th subsystem is to select the inputs $U_{i}=\left[u_{i, t}, u_{i, t+1}, \ldots, u_{i, t+N-1}\right]$ to minimize the local cost $\sum_{k=0}^{N-1} l_{i}^{P}\left(x_{i, t+k \mid t}, u_{i, t+k}, v_{i, t+k}, P\right)$. The other player for the $i$-th subsystem chooses $P_{i}=\left[p_{i, t}, p_{i, t+1}, \ldots, p_{i, t+N-1}\right]$ with the objective to maximize $\sum_{k=0}^{N-1} p_{i, t+k}^{T}\left(v_{i, t+k}-\sum_{j=1, j \neq i}^{M} A_{i j} x_{j, t+k \mid t}\right)$.

The inner decoupled optimization problems in problem P2 represent Nonlinear Programming (NLP) sub-problems, since the constraints (19) are nonlinear in the optimization variables. Each NLP sub-problem is presented as follows:

\section{Problem P3 ${ }^{i}$ (i-th NLP sub-problem):}

$$
V_{i}^{\text {opt }}\left(P, x_{i}\right)=\min _{U_{i}, X_{i}, V_{i}} \sum_{k=0}^{N-1} l_{i}^{P}\left(x_{i, t+k \mid t}, u_{i, t+k}, v_{i, t+k}, P\right)
$$

subject to $x_{i, t \mid t}=x_{i}$ and:

$$
\begin{aligned}
& x_{i, t+k \mid t} \in \mathcal{X}_{i}, k=1, \ldots, N-1 \\
& u_{i, t+k} \in \mathcal{U}_{i}, k=0,1, \ldots, N-1 \\
& x_{i, t+k+1 \mid t}=f_{i}\left(x_{i, t+k \mid t}, u_{i, t+k}\right)+v_{i, t+k}, k=0,1, \ldots, N-2
\end{aligned}
$$

Denote with $\quad U_{i}^{o p t}=\left[u_{i, t}^{o p t}, u_{i, t+1}^{o p t}, \ldots, u_{i, t+N-1}^{o p t}\right]$,

$X_{i}^{o p t}=\left[x_{i, t \mid t}^{o p t}, x_{i, t+1 \mid t}^{o p t}, \ldots, x_{i, t+N-1 \mid t}^{o p t}\right]$ and

$V_{i}^{o p t}=\left[v_{i, t}^{o p t}, v_{i, t+1}^{o p t}, \ldots, v_{i, t+N-1}^{o p t}\right]$ the optimal solution of $\mathrm{P} 3^{i}$.

\subsection{Local QP approximations of the NLP problems $P 3^{i}$}

The cost functions $\sum_{k=0}^{N-1} l_{i}^{P}\left(x_{i, t+k \mid t}, u_{i, t+k}, v_{i, t+k}, P\right)$ in the problems $\mathrm{P}^{i}, \quad i=1,2, \ldots, M$ are convex, however the constraints (25) may be non-convex in the general case. Here, we locally approximate the constraints (25) by linear constraints, leading to a quasi-nonlinear approach. Let $x_{i, t \mid t}=x_{i}^{0} \in \mathcal{X}_{i}$ be arbitrary and denote the corresponding optimal solution to the sub-problem $\mathrm{P} 3^{i}$ with:

$$
\begin{aligned}
& U_{i}^{0}=U_{i}^{\text {opt }}\left(x_{i}^{0}\right)=\left[u_{i, t}^{0}, u_{i, t+1}^{0}, \ldots, u_{i, t+N-1}^{0}\right] \\
& X_{i}^{0}=X_{i}^{\text {opt }}\left(x_{i}^{0}\right)=\left[x_{i, t \mid t}^{0}, x_{i, t+1 \mid t}^{0}, \ldots, x_{i, t+N-1 \mid t}^{0}\right] \\
& V_{i}^{0}=V_{i}^{\text {opt }}\left(x_{i}^{0}\right)=\left[v_{i, t}^{0}, v_{i, t+1}^{0}, \ldots, v_{i, t+N-1}^{0}\right]
\end{aligned}
$$

The optimal solution (26) depends on the values of the prices $P$. In section 3.3, it is described how $P$ and the solution (26) are updated iteratively. Further, Taylor series expansions of the right-hand side of constraints (25) around the point $\left(U_{i}^{0}, X_{i}^{0}, V_{i}^{0}, x_{i}^{0}\right)$ lead to the locally linear constraints:

$$
\tilde{X}_{i}^{+}=S_{X_{i}}\left(\tilde{X}_{i}-\tilde{X}_{i, 0}\right)+S_{U_{i}}\left(\tilde{U}_{i}-\tilde{U}_{i, 0}\right)+\left(\tilde{V}_{i}-\tilde{V}_{i}^{0}\right)+E_{0 i}
$$

where: $\tilde{X}_{i}=\left[x_{i, t \mid t}, \ldots, x_{i, t+N-2 \mid t}\right], \tilde{X}_{i}^{0}=\left[x_{i, t \mid t}^{0}, \ldots, x_{i, t+N-2 \mid t}^{0}\right]$

$$
\begin{aligned}
& \tilde{U}_{i}=\left[u_{i, t}, \ldots, u_{i, t+N-2}\right], \tilde{U}_{i}^{0}=\left[u_{i, t}^{0}, \ldots, u_{i, t+N-2}^{0}\right] \\
& \tilde{V}_{i}=\left[v_{i, t}, \ldots, v_{i, t+N-2}\right], \tilde{V}_{i}^{0}=\left[v_{i, t}^{0}, \ldots, v_{i, t+N-2}^{0}\right] \\
& \tilde{X}_{i}^{+}=\left[x_{i, t+1 \mid t}, \ldots, x_{i, t+N-1 \mid t}\right]
\end{aligned}
$$

Here, the matrices $S_{X_{i}}, S_{U_{i}}$, and $E_{0 i}$ are given by:

$$
\begin{aligned}
& S_{X_{i}}=\left[\begin{array}{cccc}
\nabla_{x_{i}} f_{i}\left(x_{i, t \mid t}^{0}, u_{i, t}^{0}\right) & 0_{n_{i}} & \ldots & 0_{n_{i}} \\
\vdots & & & \\
0_{n_{i}} & 0_{n_{i}} & \ldots & \nabla_{x_{i}} f_{i}\left(x_{i, t+N-2 \mid t}^{0}, u_{i, t+N-2}^{0}\right)
\end{array}\right] \\
& S_{U_{i}}=\left[\begin{array}{cccc}
\nabla_{u_{i}} f_{i}\left(x_{i, t \mid t}^{0}, u_{i, t}^{0}\right) & 0_{n_{i}, m_{i}} & \ldots & 0_{n_{i}, m_{i}} \\
\vdots & & & \\
0_{n_{i}, m_{i}} & 0_{n_{i}, m_{i}} & \ldots & \nabla_{u_{i}} f_{i}\left(x_{i, t+N-2 \mid t}^{0}, u_{i, t+N-2}^{0}\right)
\end{array}\right] \\
& E_{0 i}=\left[\begin{array}{c}
f_{i}\left(x_{i, t \mid t}^{0}, u_{i, t}^{0}\right)+v_{i, t}^{0} \\
\vdots \\
f_{i}\left(x_{i, t+N-2 \mid t}^{0}, u_{i, t+N-2}^{0}\right)+v_{i, t+N-2}^{0}
\end{array}\right]
\end{aligned}
$$

where $0_{n_{i}}$ is the $n_{i}$-dimensional square zero matrix and $0_{n_{i}, m_{i}}$ is the $n_{i} \times m_{i}$-dimensional zero matrix. It can be observed that (27) is a linear time-varying approximation of the constraints (25). Then, the NLP sub-problems $\mathrm{P} 3^{i}$ for the subsystems are approximated with the QP sub-problems:

Problem P4 ${ }^{i}$ (i-th QP sub-problem):

$$
V_{i}^{*}\left(P, x_{i}\right)=\min _{U_{i}, X_{i}, V_{i}} \sum_{k=0}^{N-1} l_{i}^{P}\left(x_{i, t+k \mid t}, u_{i, t+k}, v_{i, t+k}, P\right)
$$

subject to $x_{i, t \mid t}=x_{i},(23),(24)$, and (27).

Denote with $U_{i}^{*}=\left[u_{i, t}^{*}, \ldots, u_{i, t+N-1}^{*}\right], \quad X_{i}^{*}=\left[x_{i, t \mid t}^{*}, \ldots, x_{i, t+N-1 \mid t}^{*}\right]$ and $V_{i}^{*}=\left[v_{i, t}^{*}, \ldots, v_{i, t+N-1}^{*}\right]$ the optimal solution of problem $\mathrm{P} 4{ }^{i}$.

\section{Problem P5 (Centralized NMPC with linearized constraints):}

$$
V^{*}(x)=\min _{U} J(U, x)
$$

subject to $x_{t \mid t}=x$, constraints (7), (8), and:

$$
\begin{aligned}
& \tilde{X}_{i}^{+}=S_{X_{i}}\left(\tilde{X}_{i}-\tilde{X}_{i, 0}\right)+S_{U_{i}}\left(\tilde{U}_{i}-\tilde{U}_{i, 0}\right)+\left(\tilde{V}_{i}-\tilde{V}_{i}^{0}\right)+E_{0 i} \\
& i=1, \ldots, M
\end{aligned}
$$

where the cost function $J(U, x)$ is given by (12). Here, (34) includes the linearized constraints for all subsystems.

\section{Problem P6 (Distributed NMPC with linearized constraints):}

$$
\max _{P} \sum_{i=1}^{M} V_{i}^{*}\left(P, x_{i}\right)=\max _{P} \sum_{i=1}^{M} \min _{U_{i}, X_{i}, V_{i}} \sum_{k=0}^{N-1} l_{i}^{P}\left(x_{i, t+k \mid t}, u_{i, t+k}, v_{i, t+k}, P\right)
$$

subject to $x_{t \mid t}=x$, constraints (7), (8), (34), and:

$$
p_{t+N-1}=0
$$

Then, the decomposition of the optimization problem P5 is given by the following proposition:

\section{Proposition 1:}

Suppose that $x=\left[x_{1}, x_{2}, \ldots, x_{M}\right]$ is a feasible point for problem P5. Then:

$$
V^{*}(x)=\max _{P} \sum_{i=1}^{M} V_{i}^{*}\left(P, x_{i}\right)
$$

where maximization is subject to $p_{t+N-1}=0$.

Proof:

The proof follows similar arguments as in Giselsson and Rantzer (2010). Since the stage cost functions $l_{i}\left(x_{i, t+k \mid t}, u_{i, t+k}\right)$, 
$i=1,2, \ldots, M$ are convex, from the duality theory (Boyd and Vandenberghe (2004)) it follows that there is no duality gap between the dual problem P6 and the problem P5. The requirement $p_{t+N-1}=0$ follows from the optimality conditions of Pontryagin's principle for discrete-time systems (Bryson and Ho (1969)) and the fact that the state is not specified at the terminal time $t+N-1$. Therefore, (37) holds. Further, the maximum in (37) is attained when all elements of the gradient of $\sum_{i=1}^{M} V_{i}^{*}\left(P, x_{i}\right)$ with respect to $P$ is zero, i.e. $v_{i, t+k}^{*}-\sum_{j=1, j \neq i}^{M} A_{i j} x_{j, t+k \mid t}^{*}=0, i=1, \ldots, M, k=0,1, \ldots, N-1$. This means that the constraints (17) are satisfied at the optimum.

Proposition 1 shows that the computation of $U_{i}^{*}, X_{i}^{*}$ and $V_{i}^{*}$ for given prices $P$ is completely decentralized. However, as described in Giselsson and Rantzer (2010), finding the optimal prices requires coordination. According to the duality theory (Boyd and Vandenberghe (2004)), $V_{i}^{*}\left(P, x_{i}\right)$, $i=1, \ldots, M$ are concave functions of $P$. Therefore, the optimal price sequence $P^{*}=\left[p_{t}^{*}, p_{t+1}^{*}, \ldots, p_{t+N-1}^{*}\right]$ can be found as the limits of a gradient iteration (Giselsson and Rantzer (2010)). Given a price prediction sequence $P_{i}^{r}=\left[p_{i, t}^{r}, \ldots, p_{i, t+N-1}^{r}\right] \quad$ for the $r$-th iteration, the corresponding sequences $\quad U_{i}^{* r}=\left[u_{i, t}^{* r}, \ldots, u_{i, t+N-1}^{* r}\right]$, $X_{i}^{* r}=\left[x_{i, t \mid t}^{* r}, \ldots, x_{i, t+N-1 \mid t}^{*^{r}}\right] \quad$ and $V_{i}^{*_{r}}=\left[v_{i, t}^{* r}, \ldots, v_{i, t+N-1}^{*_{r}}\right]$ are computed locally by solving problem $\mathrm{P} 4^{i}$. Then, the prices can be updated distributedly by a gradient step:

$$
\begin{gathered}
p_{i, t+k}^{r+1}=p_{i, t+k}^{r}+\gamma_{i}^{r}\left(v_{i, t+k}^{* r}-\sum_{\substack{j=1 \\
j \neq i}}^{M} A_{i j} x_{j, t+k \mid t}^{* r}\right), k=0,1, \ldots, N-2 \\
\text { with } p_{i, t+N-1}^{r+1}=p_{i, t+N-1}^{r}=0
\end{gathered}
$$

It should be noted that Proposition 1 holds only locally, in a neighbourhood of the optimal solution $U_{i}^{0}, X_{i}^{0}, V_{i}^{0}$ to subproblems $\mathrm{P} 3^{i}, i=1,2, \ldots, M$, where the linear constraints (34) can sufficiently accurately approximate the nonlinear constraints (19). Therefore, it would be necessary to periodically update the linear constraints (34) and then to apply formula (38) for a number of steps.

\subsection{A suboptimal approach to distributed quasi-NMPC based on on-line optimization}

In Giselsson and Rantzer (2010), an approach to distributed MPC for linear systems in the absence of disturbances has been suggested, where the prices are updated according to (38). Here, a suboptimal algorithm to distributed quasi-NMPC is proposed that considers a more general class of systems, since it refers to nonlinear systems with linear couplings in the presence of bounded disturbances (see Section 2). The suggested algorithm includes two loops. In the outer loop, the NLP sub-problems $\mathrm{P}^{i}, i=1,2, \ldots, M$, are solved and the matrices of the linear constraints of the approximating QP sub-problems $\mathrm{P} 4, i=1,2, \ldots, M$ are computed. Then, in the inner loop, the price sequences and solution are updated based on Proposition 1 and applying formula (38) for a given number of steps. The algorithm is described by:

\section{Algorithm 1 (Distributed quasi-NMPC by on-line optimization):}

1. Given numbers $Q$ and $L$, step sizes $\gamma_{i}, i=1,2, \ldots, M$ and arbitrary guesses $P_{i}^{0}, i=1,2, \ldots, M$ for the price sequences. Let $t=0$.

2. Let the state at time $t$ be $x(t)=x=\left[x_{1}, \ldots, x_{M}\right]$.

3. for $q=1,2, \ldots, Q$ do

4. For $x_{i, t \mid t}=x_{i}$ compute distributedly the optimal solutions $U_{i}^{0}=U_{i}^{\text {opt }}\left(x_{i}\right), \quad X_{i}^{0}=X_{i}^{\text {opt }}\left(x_{i}\right), \quad V_{i}^{0}=V_{i}^{\text {opt }}\left(x_{i}\right) \quad$ to the NLP sub-problems $\mathrm{P}^{i}, i=1,2, \ldots, M$, corresponding to the price sequences $P_{i}^{0}=\left[p_{i, t}^{0}, \ldots, p_{i, t+N-1}^{0}\right]$. Compute the matrices $S_{X_{i}}, S_{U_{i}}$, and $E_{0 i}$ associated to the approximating QP sub-problems $\mathrm{P} 4{ }^{i}, i=1,2, \ldots, M$.

5. for $r=0,1, \ldots, L-1$ do

6. For $i$-th subsystem, $i=1,2, \ldots, M$, communicate the price $\quad P_{j}^{r}=\left[p_{j, t}^{r}, \ldots, p_{j, t+N-1}^{r}\right]$, $j=1, \ldots, M, \quad j \neq i \quad$ of the interconnected subsystems.

7. Compute the sequences $U_{i}^{*_{r}}=\left[u_{i, t}^{{ }^{r} r}, \ldots, u_{i, t+N-1}^{*_{r}}\right]$, $X_{i}^{* r}=\left[x_{i, t \mid t}^{*_{r}}, \ldots, x_{i, t+N-1 \mid t}^{* r}\right]$ and $V_{i}^{* r}=\left[v_{i, t}^{* r}, \ldots, v_{i, t+N-1}^{* r}\right]$ corresponding to the price sequence $P^{r}=\left[p_{t}^{r}, \ldots, p_{t+N-1}^{r}\right]$ by solving distributedly the QP sub-problems $\mathrm{P} 4^{i}, i=1,2, \ldots, M$.

8. For $i$-th subsystem, $i=1,2, \ldots, M$, communicate the state trajectories $X_{j}^{*_{r}}=\left[x_{j, t \mid t}^{*^{r}}, x_{j, t+1 \mid t}^{*^{r}}, \ldots, x_{j, t+N-1 \mid t}^{*_{r}}\right]$, $j=1, \ldots, M, \quad j \neq i \quad$ of the interconnected subsystems.

9. Compute distributedly the updates $P_{i}^{r+1}=\left[p_{i, t}^{r+1}, \ldots, p_{i, t+N-1}^{r+1}\right], i=1,2, \ldots, M$ of the price sequences by applying (38) for $\gamma_{i}^{r}=\gamma_{i}$, $i=1,2, \ldots, M$.

10. end

11. Let $P_{i}^{0}=P_{i}^{L}, i=1,2, \ldots, M$.

12. end

13. Apply to the overall system the control inputs $u_{i}(t)=u_{i, t}^{* L-1}, i=1,2, \ldots, M$.

14. Let $t=t+1$ and go to step 2 .

The steps 4 to 11 in Algorithm 1 include an iterative solution of the NLP sub-problems $\mathrm{P}^{i}$, approximating them with the QP sub-problems $\mathrm{P} 4^{i}$, and then updating the prices by utilizing Proposition $1^{1}$.

\footnotetext{
1 Alternatively, an approach similar to ( $\mathrm{Li}$ and Biegler (1989), Oliveira and Biegler (1995)) can be applied, where the idea would be to avoid solving the NLP sub-problems $\mathrm{P} 3^{i}$ in step 4 and to formulate the approximating QP sub-problems $\mathrm{P} 4^{i}$ by using the optimal sequences $U_{i}^{*}, X_{i}^{*}$ and $V_{i}^{*}$, computed in the previous time instant.
} 


\section{EXAMPLE}

Consider the following second order system composed of two subsystems $S_{1}$ and $S_{2}$ (Magni and Scattolini (2006)):

$$
\begin{aligned}
& S_{1}: x_{1}(t+1)=\sqrt{x_{1}(t)^{2}+1}+u_{1}(t)-1+\eta_{1} x_{2}(t)+d_{1}(t) \\
& S_{2}: x_{2}(t+1)=e^{-\sin \left(x_{2}(t)\right)}+u_{2}(t)-1+\eta_{2} x_{1}(t)+d_{2}(t)
\end{aligned}
$$

Thus, the functions $f_{i}, g_{i}, i=1,2$ in description (1) are:

$$
\begin{aligned}
& f_{1}\left(x_{1}(t), u_{1}(t)\right)=\sqrt{x_{1}(t)^{2}+1}+u_{1}(t)-1 \\
& f_{2}\left(x_{2}(t), u_{2}(t)\right)=e^{-\sin \left(x_{2}(t)\right)}+u_{2}(t)-1 \\
& g_{1}(x(t))=\eta_{1} x_{2}(t), \quad g_{2}(x(t))=\eta_{2} x_{1}(t)
\end{aligned}
$$

The functions $g_{i}$ satisfy Assumption A4 and they describe the mutual influence of the two subsystems. The disturbances are assumed to be the states of the following asymptotically stable first order systems (Magni and Scattolini (2006)):

$$
d_{i}(t+1)=0.9 d_{i}(t), i=1,2
$$

and they are bounded by $\left|d_{i}(t)\right| \leq 1, i=1,2$. The following constraints are imposed on the system (41):

$$
\begin{aligned}
& -0.3 \leq u_{i}(t) \leq 0.5, i=1,2 \\
& -2 \leq x_{1}(t) \leq 2, \quad-1.5 \leq x_{2}(t) \leq 1.5
\end{aligned}
$$

The coefficients related to the couplings between the two subsystems are $\eta_{1}=\eta_{2}=0.4$. The prediction horizon is $N=5$ and the weighting matrices are $Q_{i}=R_{i}=1, i=1,2$. The centralized NMPC problem is represented as a distributed NMPC problem (problem P6) by applying the dual decomposition approach. Then, Algorithm 1 with parameters $Q=5, L=3, \gamma_{i}=0.3, i=1,2$ is used to generate the two control inputs for an initial state $x(0)=\left[\begin{array}{ll}0.3 & 0.3\end{array}\right]$ and initial disturbances $d(0)=\left[\begin{array}{ll}1 & 1\end{array}\right]$. The corresponding trajectories of the prices $p_{1}, p_{2}$, the control inputs $u_{1}, u_{2}$, the states $x_{1}, x_{2}$ and the disturbances $d_{1}, d_{2}$ associated to the two subsystems are depicted in Fig. 1 to Fig. 6. The trajectories obtained with the following approaches are compared:

- The suboptimal distributed NMPC approach with linearization of the nonlinear constraints (25) (described in section 3);

- A suboptimal distributed NMPC approach without linearization of the nonlinear constraints (25). In this case, a modification of Algorithm 1 is used for the on-line computation of the control inputs. It has only one loop, where the optimal solutions of the NLP sub-problems P3 ${ }^{i}$, $i=1,2, \ldots, M$ are computed distributedly, and then the price sequences are updated by applying (38) by using the computed optimal solutions. The loop is repeated $Q=5$ times and the step size in (38) is $\gamma_{i}=0.3, i=1,2$.

- The exact distributed NMPC approach, which solves problem $\mathrm{P} 6$ at each time instant.

- The centralized NMPC approach, which solves problem $\mathrm{P} 1$ at each time instant.

The computational complexity of both suboptimal distributed NMPC approaches is compared to that of the exact distributed NMPC approach and the centralized NMPC approach. For this aim, the respective trajectories are determined for 100 initial states, obtained by gridding the state space $[-1,1] \times[-1,1]$. The results are presented in Table 1 , where also the possibility of these approaches to find a feasible solution is compared and expressed in terms of percentage from the total number of initial states. The computations are performed on a $3 \mathrm{GHz}$ Intel Core 2 Duo processor.
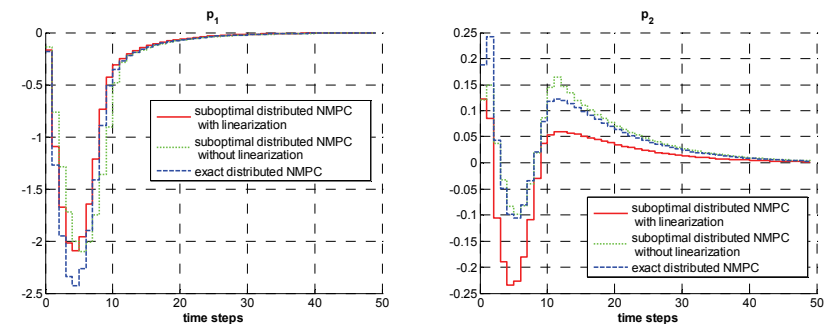

Fig. 1. The prices $p_{1}$ and $p_{2}$.

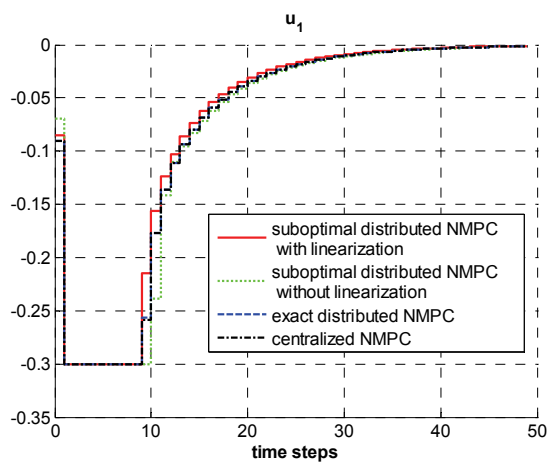

Fig. 2. The control input $u_{1}$ for subsystem $S_{1}$.

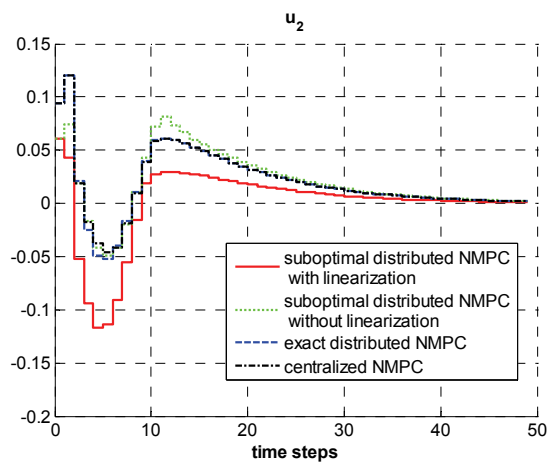

Fig. 3. The control input $u_{2}$ for subsystem $S_{2}$.

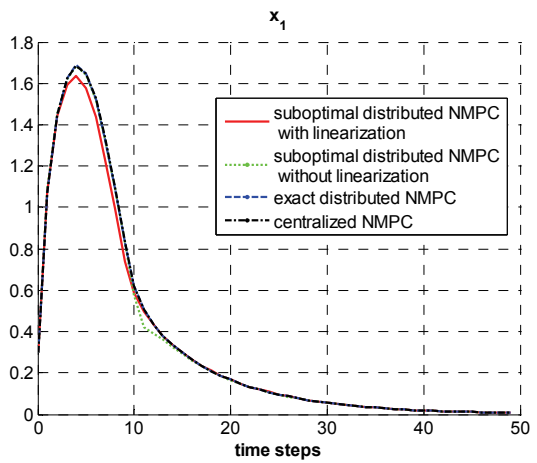

Fig. 4. The state $x_{1}$ of subsystem $S_{1}$. 


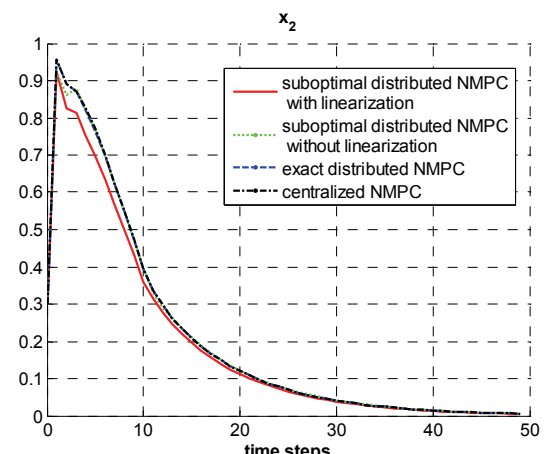

Fig. 5. The state $x_{2}$ of subsystem $S_{2}$.

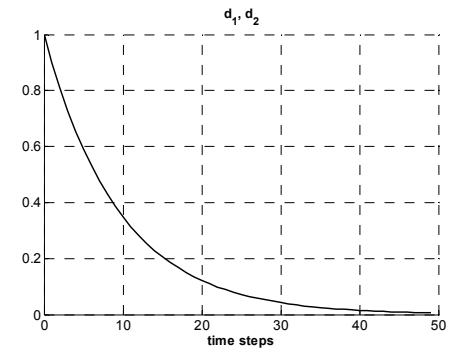

Fig. 6. The disturbances $d_{1}, d_{2}$.

Table 1. Comparison of different NMPC approaches.

\begin{tabular}{||cccc||}
\hline Method & $\begin{array}{c}\text { Percentage } \\
\text { of feasible } \\
\text { solutions }\end{array}$ & $\begin{array}{c}\text { Average } \\
\text { CPU time } \\
{[\mathrm{s}]}\end{array}$ & $\begin{array}{c}\text { Maximal } \\
\text { CPU time } \\
{[\mathrm{s}]}\end{array}$ \\
\hline $\begin{array}{c}\text { Suboptimal distributed } \\
\text { NMPC without } \\
\text { linearization }\end{array}$ & $79 \%$ & 0.66 & 0.74 \\
\hline $\begin{array}{c}\text { Suboptimal distributed } \\
\text { NMPC with } \\
\text { linearization }\end{array}$ & $79 \%$ & 1.81 & 1.86 \\
\hline $\begin{array}{c}\text { Exact } \text { distributed } \\
\text { NMPC }\end{array}$ & $79 \%$ & 10.28 & 14.86 \\
\hline Centralized NMPC & $28 \%$ & 0.66 & 2.30 \\
\hline \hline
\end{tabular}

It can be seen from Table 1 that the suboptimal distributed NMPC approach without linearization of the nonlinear constraints is the most computationally efficient approach. Both suboptimal approaches are more efficient in comparison to the exact distributed NMPC approach. A disadvantage of the centralized approach is that it fails to find a feasible solution for a significant number of initial states (in this sense it is about three times less efficient than the distributed NMPC approaches). A possible reason for this is the fact that in the presence of disturbances, the worst-case state constraints related to both subsystems (constraints (7) in the problem P1) can be difficult to be satisfied at the same time for some of the initial states. In contrast, the distributed NMPC approaches lead to two completely decentralized NMPC problems, where worst-case disturbance assumptions of only the individual subsystems are made (not simultaneously).

\section{ACKNOWLEDGEMENTS}

This work was sponsored by the Research Council of Norway through the Strategic University Program on Control, Information and Communication Systems for Environmental and Safety-Critical Systems.

\section{REFERENCES}

Arrow, K.J., Hurwicz, L., and Uzawa, H. (1958). Studies in linear and nonlinear programming, Stanford Univ. Press.

Boyd, S. and Vandenberghe, L. (2004). Convex optimization, Cambridge University Press.

Bryson, A. and Ho, Y. (1969). Applied optimal control: Optimization, estimation and control, Blaisdell Publ. Co., Waltham, Massachusetts.

Cohen, G. and Miara, B. (1990). Optimization with an auxiliary constraint and decomposition. SIAM Journal on Control and Optimization, 28, 137-157.

Constantinides, G.A. (2009). Parallel architectures for model predictive control. In Proc. European Control Conference, Budapest, Hungary, pp. 138-143.

Dantzig, G.B. and Wolfe, P. (1961). The decomposition algorithm for linear programs. Econometrica, 29, 767778.

Dunbar, W.B. and Murray, R.M. (2006). Distributed receding horizon control for multi-vehicle formation stabilization. Automatica, 42, 549-558.

Giselsson, P. and Rantzer, A. (2010). Distributed model predictive control with suboptimality and stability guarantees. In Proc. Conference on Decision and Control, Atlanta, GA, pp. 7272-7277.

Keviczky, T., Borrelli, F., and Balas, G.J. (2006). Decentralized receding horizon control for large scale dynamically decoupled systems. Automatica, 42, 21052115.

Kim, T.H. and Sugie, T. (2005). Robust decentralized MPC algorithm for a class of dynamically interconnected constrained systems. In Proc. Conference on Decision and Control, Seville, Spain, pp. 290-295.

Li, W.C. and Biegler, L.T. (1989). Multistep, Newton-type control strategies for constrained nonlinear processes. Chem. Eng. R\& Des., 67, 562-577.

Magni, L. and Scattolini, R. (2006). Stabilizing decentralized model predictive control of nonlinear systems. Automatica, 42, 1231-1236.

Oliveira, N.M.C. and Biegler, L.T. (1995). An extension of Newton-type algorithms for nonlinear process control. Automatica, 31, 281-286.

Rantzer, A. (2007). On prize mechanisms in linear quadratic team theory. In Proc. Conference on Decision and Control, New Orleans, pp. 1112-1116.

Rantzer, A. (2009). Dynamic dual decomposition for distributed control. In Proc. American Control Conference, St. Louis, MO, USA, pp. 884-888.

Scattolini, R. (2009). Architectures for distributed and hierarchical Model Predictive Control - A review. Journal of Process Control, 19, 723-731.

Venkat, A.N., Rawlings, J.B., and Wright, S.J. (2004). Plantwide optimal control with decentralized MPC. In Proc. IFAC Conference on Dynamics and Control of Process Systems, Boston, MA.

Zhang, Y. and Li, S. (2007). Networked model predictive control based on neighbourhood optimization for serially connected large-scale processes. Journal of Process Control, 17, 37-50. 